\title{
Constraints on the antistar fraction in the Solar system neighborhood from the 10-years Fermi Large Area Telescope gamma-ray source catalog
}

\author{
Simon Dupourqué, ${ }^{a, *}$ Luigi Tibaldo ${ }^{a}$ and Peter von Ballmoos ${ }^{a}$ \\ ${ }^{a}$ Institut de Recherche en Astrophysique et Planétologie, \\ 9 Avenue du Colonel Roche, Toulouse, France \\ E-mail: sdupourque@irap.omp.eu
}

It is generally taken for granted that our Universe is free of antimatter objects and domains. This certitude has recently been challenged by the possible detection of anti-helium nuclei by AMS-02. Should the observation be confirmed, the existence of nearby antistars would make a plausible hypothesis to explain the origin of the antinuclei. In this paper we use the 10-years Fermi Large Area Telescope (LAT) gamma-ray source catalog to set constraints on the abundance of antistars around the Sun. We identify in the catalog 14 antistar candidates not associated with any objects belonging to established gamma-ray source classes and with a spectrum compatible with baryon-antibaryon annihilation. We use them along with an estimate of the LAT sensitivity to antistars to set upper limits on the local antistar fraction $f_{\bar{*}}$ with respect to normal stars. We provide parametric limits as a function of the closest antistar mass, velocity, and surrounding matter density. We also employ a novel Monte Carlo method to set limits for a few hypotheses about the antistar population. For a population with properties equivalent to those of regular stars concentrated in the Galactic disk we obtain $f_{\bar{*}}<2.5 \times 10^{-6}$ at $95 \%$ confidence level, which is 20 times more constraining than limits previously available. For a primordial population of antistars distributed in the Galactic halo we obtain new local upper limits which decrease as a function of antistar mass $M$ from $f_{\bar{*}}<0.2$ at $95 \%$ confidence level for $M=1 M_{\odot}$ to $f_{\bar{*}}<1.6 \times 10^{-4}$ at $95 \%$ confidence level for $M=10 M_{\odot}$. By combining these limits with existing microlensing constraints for lighter objects in the Magellanic clouds, we infer that a primordial population of halo antistars must have a density lower than $O\left(10^{-5} \mathrm{pc}^{-3}\right)$ to $O\left(10^{-2} \mathrm{pc}^{-3}\right)$ depending on their masses. Our limits can constrain models for the origin and propagation of antinuclei in cosmic rays.

$37^{\text {th }}$ International Cosmic Ray Conference (ICRC 2021)

July 12th - 23rd, 2021

Online - Berlin, Germany

\footnotetext{
${ }^{*}$ Presenter
} 


\section{Introduction}

We generally take it for granted that equal amounts of matter and antimatter were produced in the Big Bang, yet the observable Universe seems to contain only negligible quantities of antimatter. Baryonic antimatter in our Solar and Galactic neighborhood can be constrained by the observation of high-energy gamma rays [20]: when coming into contact with normal matter, it would produce annihilation radiation featuring a characteristic spectrum peaking around half the mass of the neutral pion at $\sim 70 \mathrm{MeV}$, and with a cutoff around the mass of the proton at $938 \mathrm{MeV}$ [5]. The non detection of this annihilation feature in gamma rays has virtually excluded the existence of substantial amounts of baryonic antimatter in the solar system, the solar neighborhood, the Milky Way, and up to the scale of galaxy clusters [20,21,24]. When combined with observations of the largely isotropic Cosmic Microwave Background, the lack of an "MeV-bump" in gamma-rays has led to the presently accepted paradigm in which a matter-antimatter symmetric Universe can be ruled out [9].

However, the standard paradigm that our local Universe is completely matter dominated has recently been challenged by the tentative detection of a few anti-helium nuclei by the Alpha Magnetic Spectrometer experiment (AMS-02) on the International Space Station [22]. AMS-02 measures roughly one anti-helium in a hundred million helium. Amongst the eight anti-helium events reported, six are compatible with being anti-helium-3 and two with anti-helium-4. Several authors, e.g., Salati et al. [18], had pointed out that "the detection of a single anti-helium [... ] would be a smoking gun [...] for the existence of antistars and of antigalaxies". As discussed since Steigman [20] and recently remarked by Poulin et al. [15], gamma-ray observations can be used to constrain the abundance of nearby antistars. Therefore, in this paper we use the recently published $4^{\text {th }}$ catalog of high-energy gamma-ray sources detected with the Fermi Large Area Telescope (LAT) data release 2 (4FGL-DR2) [3, 6] to derive constraints on the existence of antistars in the solar neighborhood. All the results presented here are detailed and discussed in Dupourqué et al. [11].

\section{Constraining antistars with 4FGL-DR2}

\subsection{Antistar candidates in 4FGL-DR2}

4FGL-DR2 [3, 6] is based on 10 years of observations with the LAT in the energy range from $50 \mathrm{MeV}$ to $1 \mathrm{TeV}$. It contains 5787 gamma-ray sources with their spectral parameters, spectral energy distributions, light curves, and multiwavelength associations. We select antistar candidates in $4 \mathrm{FGL}-\mathrm{DR} 2{ }^{1}$ based on the following criteria:

- extended sources are excluded since the angular size of a star is several orders of magnitude smaller than the LAT resolution at low energy, thus antistars are expected to be point-like sources;

- sources associated with objects known from other wavelengths that belong to established gamma-ray source classes (e.g., pulsars, active galactic nuclei) are excluded;

\footnotetext{
${ }^{1}$ We used the initial release of the catalog (file gll_psc_v23.fit), but we checked that all results are unchanged for the latest version available at the moment of writing which includes more optical classifications (file gll_psc_v26. fit).
} 


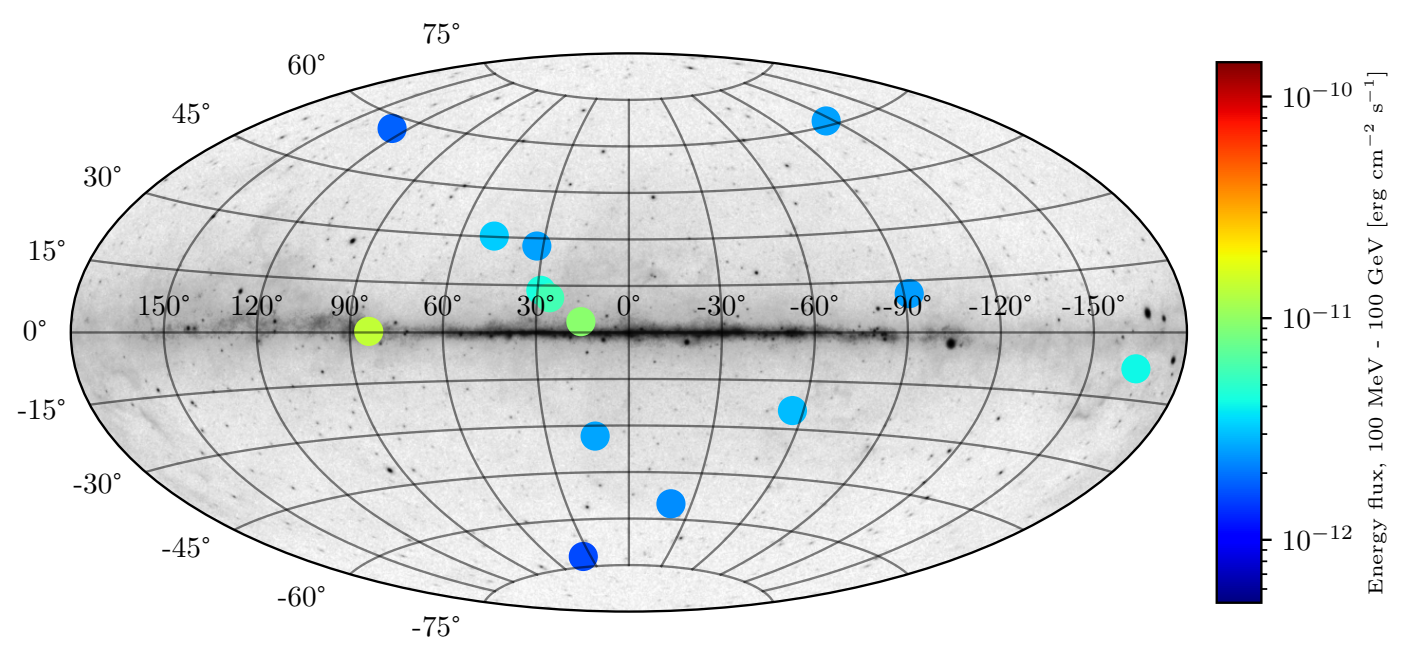

Figure 1: Positions and energy flux in the $100 \mathrm{MeV}-100 \mathrm{GeV}$ range of antistar candidates selected in 4FGL-DR2. Galactic coordinates. The background image shows the Fermi 5-year all-sky photon counts above $1 \mathrm{GeV}$ (Image credit: NASA/DOE/Fermi LAT Collaboration)

- sources with emission detected at $>3 \sigma$ above $1 \mathrm{GeV}$ are excluded since the emission spectrum from proton-antiproton annihilation is null above $938 \mathrm{MeV}$ (mass of the proton); to our knowledge this is the first time that spectral criteria are used to select candidate antistars in gamma-ray catalogs;

- sources flagged in the catalog as potential spurious detections related to uncertainties in the background models or nearby bright sources (flags 1 to 6 ) are excluded.

This results in 14 antistar candidates, Figure 1 shows their positions in the sky and fluxes. They do not follow a particular pattern on the sky, and they are all faint and close to the LAT detectability threshold. The nature of these sources cannot be firmly established at present. Besides the tentative antistar interpretation, they may be sources belonging to a known gamma-ray source class, such as pulsars or active galactic nuclei, that could be identified by searching for periodicity in gamma-ray [e.g., 2] and radio data [e.g., 13], or for spectral signatures in optical and infrared observations [e.g., 14], respectively. Furthermore, they may also correspond to imperfections of the background interstellar emission model, e.g, owing to limitations of ISM tracers, for which improvements can be achieved thanks to multiwavelength data. In the following we will use the candidate list to set upper limits on the antistar abundance in the region around the Sun.

\subsection{Sensitivity to an antistar signal}

In order to use the candidates to constrain the population of antistars in the solar neighborhood we need to establish the sensitivity to an antistar signal in 4FGL-DR2. 4FGL-DR2 sources were selected based on the criterion that TS $\geq 25$ over the entire energy band from $50 \mathrm{MeV}$ to $1 \mathrm{TeV}$ (detection significance $\geq 4.1 \sigma$ ). Thus, to determine whether an antistar would appear in the catalog, we need to determine the minimum flux that it must have so that its $\mathrm{TS}=25$. 


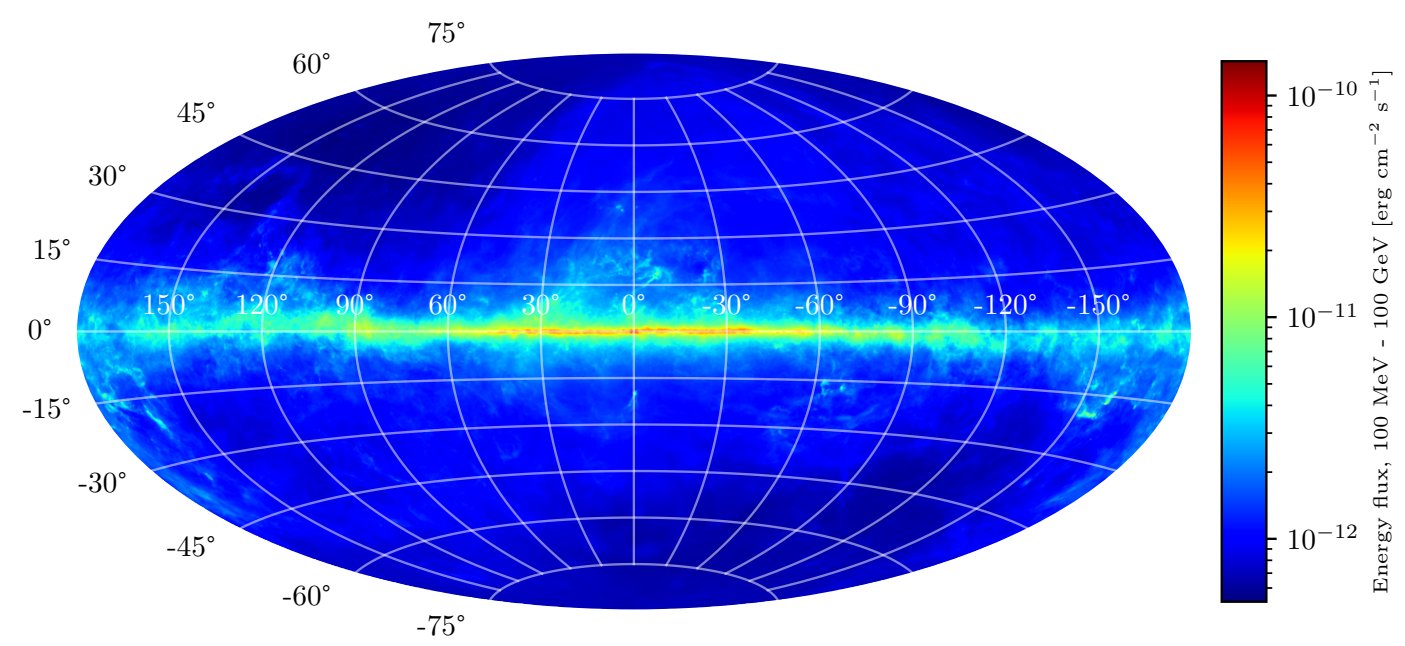

Figure 2: Minimum energy flux in the $100 \mathrm{MeV}-100 \mathrm{GeV}$ energy range for a pointlike source with matter-antimatter annihilation spectrum to be detectable in the 4FGL-DR2 catalog. Galactic coordinates.

To do so, we follow the method proposed in Appendix A of the first Fermi-LAT source catalog (1FGL) [1]. This consists in calculating semi-analytically TS for a pointlike source based on the Instrument Response Functions (IRFs) of the LAT, the spectrum of the source $S(E)$ and the background model $B(E)$. The LAT sensitivity to an antistar signal can be expressed in the form of a sky map, where each pixel represents the flux necessary to obtain $\mathrm{TS}=25$ for a pointlike source with a matter-antimatter annihilation spectrum at this position. The resulting sky map is shown in Figure 2. Since the main background is given by Galactic interstellar emission, as expected antistars would be more easily observed outside the Galactic plane, which tends to be the case for our candidates.

\section{The fraction of antistars in the solar system neighborhood}

The limits on the antistar population in the solar neighborhood are established based on the hypothesis that antistars in the Galaxy would accrete matter from the ISM with subsequent $p-\bar{p}$ annihilation at their surface [20]. Following the steps of Steigman [20], we compute the total luminosity of an antistar for Bondi-Hoyle-Littleton accretion [12] and using the gamma-ray yield per $p-\bar{p}$ annihilation from Backenstoss et al. [5]. Taking into account explicitly the speed of sound $c$ and the density of matter $\rho$ in the ISM, this yields

$$
L_{\gamma}=8.45 \times 10^{35}\left(\frac{\rho}{\mathrm{m}_{p} \mathrm{~cm}^{-3}}\right)\left(\frac{M}{M_{\odot}}\right)^{2}\left(\frac{\sqrt{v^{2}+c^{2}}}{10 \mathrm{~km} \mathrm{~s}^{-1}}\right)^{-3}\left[\mathrm{ph} \mathrm{s}^{-1}\right] .
$$

\subsection{Parametric derivation of the antistar fraction}

In a first time, we establish limits on the antistar fraction based on the method proposed by Steigman [20]. The method consists in assuming that the brightest antistar candidate is the nearest antistar. One can thus determine its distance based on its photon flux $\Phi_{\max }$ for any mass, velocity, 

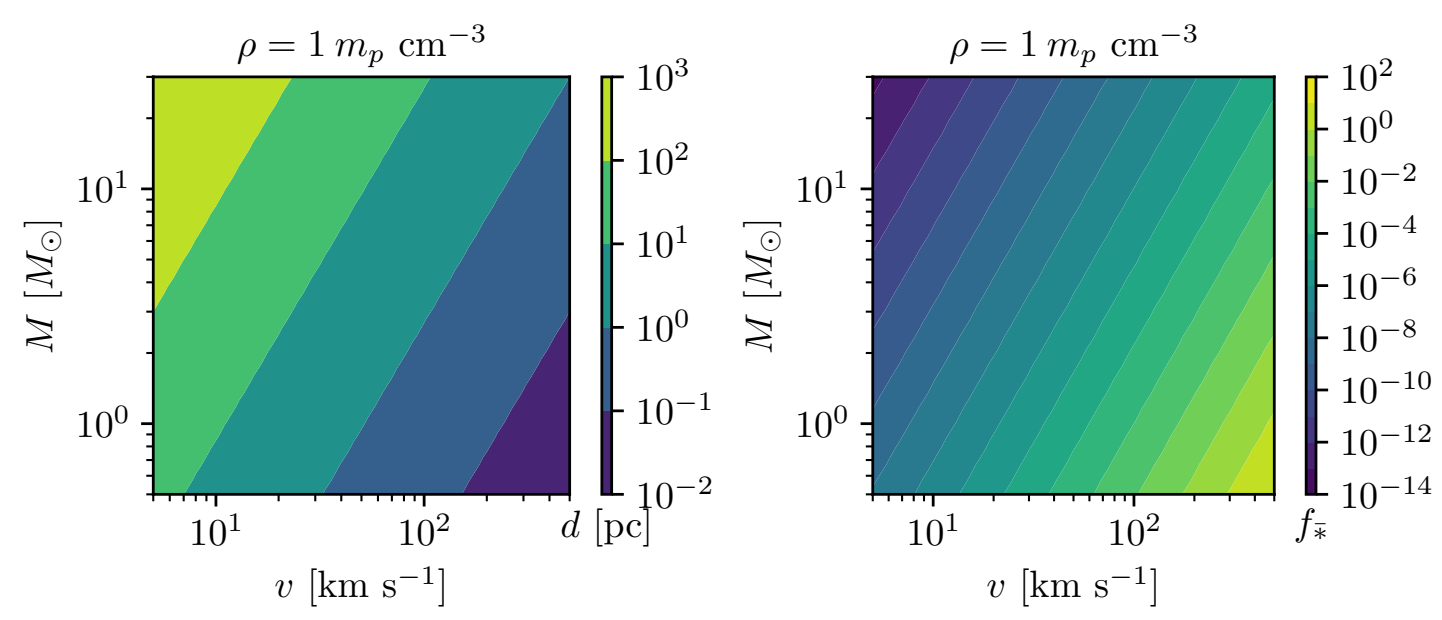

Figure 3: Left: distance $d$ of the closest antistar candidate in the 4FGL-DR2 catalog based on the luminosity relation in Equation 1. Right: corresponding upper limit on the antistar fraction $f_{\bar{*}}$ from Equation 2. In both panels the quantities are shown as a function of velocity $v$ w.r.t. the ISM and antistar mass $M$ for an ISM density $\rho=1 m_{p} \mathrm{~cm}^{-3}$.

and ISM density values. The sphere with radius equal to such distance is assumed to contain at most one antistar, and the fraction of antistars to normal stars is given by $f_{\bar{*}}=\left(n_{*} V\right)^{-1}$ where $n_{*}$ is the local star density, and $V$ is the volume of the sphere. In parametric form, the antistar fraction upper limit is given by

$$
f_{\bar{k}} \leq 2.68 \times 10^{3}\left(\frac{\Phi_{\max }}{\mathrm{cm}^{-2} \mathrm{~s}^{-1}}\right)^{3 / 2}\left(\frac{\rho}{\mathrm{m}_{p} \mathrm{~cm}^{-3}}\right)^{-3 / 2}\left(\frac{M}{M_{\odot}}\right)^{-3}\left(\frac{\sqrt{v^{2}+c^{2}}}{10 \mathrm{~km} \mathrm{~s}^{-1}}\right)^{9 / 2}
$$

We obtain the minimal distances and upper limits on the antistar fraction shown in in Figure 3 as a function of antistar mass and velocity for an ISM density of $\rho=1 \mathrm{~m}_{p} \mathrm{~cm}^{-3}$. This method for estimating $f_{\bar{*}}$ has several limitations: it relies on arbitrary choices for the parameters and the obtained limits do not have a well-defined statistical meaning. In addition, Equation 2 takes into account the flux of one source only, neglecting the rest of the exploitable information.

\subsection{Monte Carlo derivation of the antistar fraction}

\subsubsection{General method description}

To overcome the limitations of the previous procedure, we propose a novel Monte Carlo method. The method relies on a well-defined hypothesis on the antistar population with only one free parameter (the antistar fraction $f_{\bar{*}}$ or the antistar density $n_{\bar{*}}$ ). Based on this hypothesis we build an estimator $\hat{N}_{\bar{*}}$ for the number of antistars that should be detected for a given value of the free parameter. For each parameter value, we generate 1000 synthetic antistar populations according to the hypothesis and calculate the associated gamma-ray fluxes. The fluxes are then compared to the sensitivity map (Figure 2) to check whether the synthetic sources would be detected or not, and determine the number of expected detections. We determine the value of the free parameters that yields $\hat{N}_{\bar{*}} \leq 14$ for $95 \%$ of the synthetic populations, and $\hat{N}_{\bar{*}}>14$ for $5 \%$ of the populations, where 

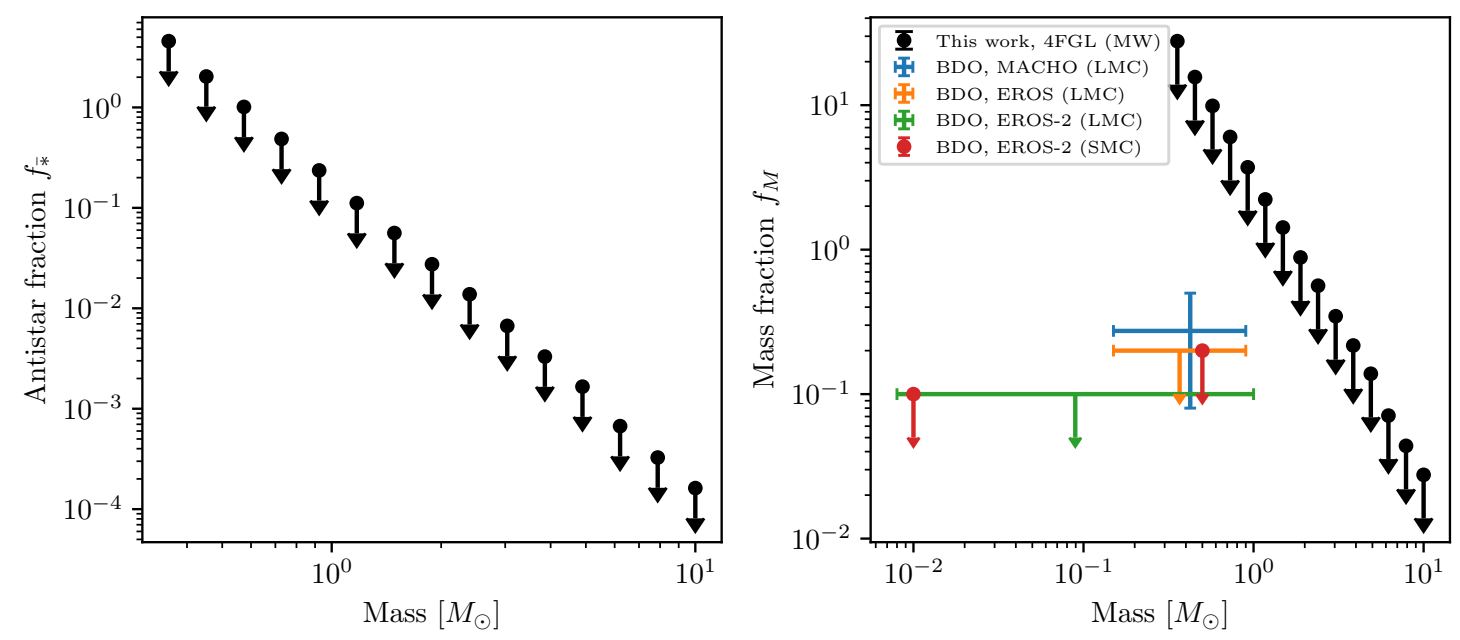

Figure 4: Antistar fraction (left) and mass fraction (right) upper limits as a function of antistar mass obtained from 4FGL-DR2 under the hypothesis that antistars are primordial objects evolving in the Milky Way (MW) halo (see text for details). In the right panel we compare gamma-ray constraints on antistars to earlier results from microlensing experiments on BDOs for the Large Magellanic Cloud (LMC) and Small Magellanic Cloud (SMC).

14 is the number of antistar candidates found in 4FGL-DR2 (Section 2.1). This provides a 95\% confidence level upper limit on the parameter value.

\subsubsection{Antistar fraction for a young disk population}

The first hypothesis that we consider is that antistars have the same properties as normal stars, dominated by the young stellar populations in the Galactic disk. Although difficult to justify physically, this hypothesis makes it possible to compare our results with previous works that employ star-like parameters for antistars. In order to generate synthetic star population we use the code Galaxia [19], which implements the state-of-the-art Besançon model [17].

Using this method, the local fraction of antistars is estimated at $f_{\bar{*}}<2.5 \times 10^{-6}$ at $95 \%$ confidence level. This result is 20 times more constraining than the limit reported based on 2FGL [24], and no longer relies on arbitrary choices for the antistar properties. For starlike properties the antistars more likely detected by the LAT would have masses $\sim 1 M_{\odot}$, velocities w.r.t. the ISM of $\sim 10 \mathrm{~km} \mathrm{~s}^{-1}$, and distances of $\sim 500 \mathrm{pc}$. Stars with masses $<1 M_{\odot}$, although more abundant, are not efficient enough in terms of accretion to be predominant in the detected sources. Velocity is a key component, it is mainly the speed of an antistar that will determine the maximum distance at which it can be observed. Stars of high mass and especially of low velocity are the most distant objects observable by the LAT, up to $10 \mathrm{kpc}$.

\subsubsection{Antistar fraction for a primordial halo population}

A more physically-motivated scenario discussed in the literature is that antistars may be primordial objects produced in the early Universe, e.g., in the Affleck-Dine scenario for baryogenesis $[7,10]$. Under this hypothesis, antistars would now be present in galactic halos as a subclass of pri- 
mordial baryon-dense objects (BDOs). The contribution of BDOs to halo masses was constrained so far through microlensing observations, most recently in the Magellanic Clouds by MACHO [4], EROS [16], and EROS-2 [8, 23]. We test this scenario against the 4FGL-DR2 antistar candidates by using our Monte Carlo method. Blinnikov et al. [7] provide a typical velocity for primordial antistars of $500 \mathrm{~km} \mathrm{~s}^{-1}$. We generate mock antistar populations with uniform spatial distribution and a velocity of $500 \mathrm{~km} \mathrm{~s}^{-1}$ with isotropic distribution. As we lack clear model prescriptions for the mass distribution, we repeat the procedure several times for fixed mass values in the range from $0.3 M_{\odot}$ to $10 M_{\odot}$. The mass range is chosen to compare with earlier results from microlensing.

The resulting antistar fraction $f_{\bar{*}}$ and mass fraction $f_{M}$ w.r.t the local dark-matter density are shown in Figure 4. Gamma rays provide novel constraints for the mass range $\gtrsim 1 M_{\odot}$, while for smaller masses microlensing constraints from nearby galaxies remain stronger.Owing to their large speed, and therefore low accretion rates, primordial antistars can be detected by the LAT only at very limited distances from the Sun $(d<70 \mathrm{pc})$, and for low masses the gamma-ray upper limits are so weak that they exceed the number of observed stars.

\section{References}

[1] Abdo, A. A., Ackermann, M., Ajello, M., et al. 2010, The Astrophysical Journal Supplement Series, 188,405

[2] Abdo, A. A., Ackermann, M., Ajello, M., et al. 2009, Science, 325, 840-844

[3] Abdollahi, S., Acero, F., Ackermann, M., et al. 2020, The Astrophysical Journal Supplement Series, 247, 33

[4] Alcock, C., Allsman, R. A., Alves, D. R., et al. 2000, The Astrophysical Journal, 542, 281

[5] Backenstoss, G., Hasinoff, M., Pavlopoulos, P., et al. 1983, Nuclear Physics B, 228, 424

[6] Ballet, J., Burnett, T. H., Digel, S. W., \& Lott, B. 2020, Fermi Large Area Telescope Fourth Source Catalog Data Release 2

[7] Blinnikov, S. I., Dolgov, A. D., \& Postnov, K. A. 2015, Phys. Rev. D, 92, 023516

[8] Calchi Novati, S., Mirzoyan, S., Jetzer, P., \& Scarpetta, G. 2013, Monthly Notices of the Royal Astronomical Society, 435, 1582-1597

[9] Cohen, A. G., De Rújula, A., \& Glashow, S. L. 1998, The Astrophysical Journal, 495, 539

[10] Dolgov, A. D. \& Blinnikov, S. I. 2014, Physical Review D, 89, 021301(R)

[11] Dupourqué, S., Tibaldo, L., \& von Ballmoos, P. 2021, Phys. Rev. D, 103, 083016

[12] Edgar, R. 2004, New Astronomy Reviews, 48, 843

[13] Keith, M. J., Johnston, S., Ray, P. S., et al. 2011, Monthly Notices of the Royal Astronomical Society, 414, 1292-1300 
[14] Peña-Herazo, H. A., Amaya-Almazán, R. A., Massaro, F., et al. 2020, Astronomy and Astrophysics, 643, A103

[15] Poulin, V., Salati, P., Cholis, I., Kamionkowski, M., \& Silk, J. 2019, Physical Review D, 99, 023016

[16] Renault, C., Afonso, C., Aubourg, E., et al. 1997, Astronomy and Astrophysics, 324, L69

[17] Robin, A. C., Reylé, C., Derrière, S., \& Picaud, S. 2003, Astronomy and Astrophysics, 409, 523

[18] Salati, P., Chardonnet, P., \& Orloff, J. 1999, Nuclear Physics B - Proceedings Supplements, 70, 492 , proceedings of the Fifth International Workshop on topics in Astroparticle and Underground Physics

[19] Sharma, S., Bland-Hawthorn, J., Johnston, K. V., \& Binney, J. 2011, The Astrophysical Journal, 730,3

[20] Steigman, G. 1976, Annual Review of Astronomy and Astrophysics, 14, 339

[21] Steigman, G. 2008, Journal of Cosmology and Astroparticle Physics, 2008, 001

[22] Ting, S. 2018

[23] Tisserand, P., Le Guillou, L., Afonso, C., et al. 2007, Astronomy and Astrophysics, 469, 387

[24] von Ballmoos, P. 2014, Hyperfine Interactions, 228, 100 